\title{
Transfer Matrices of Single Volute Centrifugal Pumps
}

\author{
Fabrice Carta, Jacques Charley and Guy Caignaert \\ Laboratoire de M9 canique de Lille, ENSAM 8 Bd. Louis XIV F-59046 Lille, France
}

(Received 20 March 2000; accepted 21 June 2000)

\begin{abstract}
Over the last few years, many studies have been carried out in order to analyse the hydroacoustic behaviour of hydraulic systems in the low frequency range. With that aim in view, Dumont reported that the manufacturer as well as the pump user needs to know the transfer matrix and the associated source terms ${ }^{1}$ with maximum accuracy. Most of the time, the well-known two-dimensional matrix model is used to perform pressure and flow-rate fluctuation evolutions without taking into account the vibrations of the structure. For some components of hydraulic circuits, such as rotodynamic pumps in various operating conditions, this determination of the transfer matrix can only be accurately performed with the help of experimental investigations. The experimental methodologies, developed over many years, are now well established. They are used in applications for fans and IC-engine exhaust and inlet systems. ${ }^{2,3}$ If we look at the transfer matrix of a pump, we notice that the transfer matrix is apparently independent of the operating conditions, as long as no cavitation appears in the pump. It also appears that such a transfer matrix can be determined in standstill conditions, which is obviously very convenient as experiments become quicker and cheaper. This paper presents a few arguments about the quality criteria of such experimental devices. On the basis of experimental results, obtained with a single volute centrifugal pump tested in air, some transfer matrix characteristics are shown in order to know when the pump can be considered as a reciprocal or a quasi-reciprocal system according to Brennen's classification. ${ }^{4}$
\end{abstract}

\section{INTRODUCTION}

Today, operating machinery that radiates noise can be controlled quite efficiently. However, it proves difficult to eliminate or to reduce the hydroacoustic noise linked to structure vibrations and to turbulent flows, especially within the complex geometry of hydraulic network components.

The generated acoustic plane waves, in the low frequency range, which propagate everywhere in a circuit, interact with the structure by exciting its various modes. As a major consequence, installations may be damaged when the acoustical and mechanical modes mutually intensify especially for low frequencies. Consequently, it is very useful to be able to produce models of the various phenomena in order to be able to predict such behaviour.

Up to now, attempts with three-dimensional finite elements remain difficult to implement and often require huge calculation resources for results which are still too inaccurate. The flow complexities in a turbopump lead to some weaknesses in numerical models created to represent correctly the reality of the physical phenomena. Improving the models requires taking into account the 3D and unsteady structures of the flow. In order to validate properly the results obtained with such computer codes, experimental data bases are necessary.

As numerical models of the acoustic propagation and transfer phenomena in the circuit components require a knowledge of the evolution of pressure and that of the fluctuating flow rate characteristics, our work uses a simplified black-box formulation with transfer matrices. Tests have been performed on the test rig VENTIL of the "Laboratoire de M $[$ canique de Lille", for the study in air of a single volute radial flow pump. ${ }^{5}$
Up to now, the hydroacoustic characterisation of pumps as well as centrifugal fans can only be achieved thanks to several experimental tests. For each operating point of the pump tested, at least three independent tests (from an hydroacoustic point of view) must be carried out in order to get the hydroacoustic transfer matrix and the associated sources terms; see, e.g. references ${ }^{6-8}$.

The objective of the research presented in this paper is to determine the hydroacoustic transfer matrix of a single volute centrifugal pump, tested in air in various operating conditions. Some properties of the measured transfer matrix, which can be related to the quality of measurements, are further revealed to show if the pump can be considered as a reciprocal or quasi-reciprocal hydraulic component.

The results show that, in a large frequency range, the transfer matrix appears to be nearly independent of the operating conditions. It is also demonstrated that tests in nonoperating conditions, can be used, in order to get a good estimation of the transfer matrix in the frequency range under investigation and in no-cavitating conditions.

\section{THE TEST RIG VENTIL}

The test rig VENTIL was designed in order to determine the transfer matrix in non-operating conditions, and in various operating conditions. ${ }^{\mathbf{9}, 10}$

The test set-up used in operating conditions was identical to that in non-operating conditions. However a modification of the installation proved necessary: a box with an orifice plate was located at the inlet of the suction pipe. It enables the control and measurement of the flow rate in the operating conditions, but also flow homogenisation at the impeller inlet with a honeycomb filter. 